\title{
Comparison of in vitro fertilization outcomes in ICSI cycles after human sperm preparation by density gradient centrifugation and direct micro swim-up without centrifugation
}

Simone Palini ${ }^{1}$, Silvia De Stefani ${ }^{1}$, Mariangela Primiterra ${ }^{1}$, Serena Benedetti ${ }^{2}$, Stefano Barone ${ }^{3}$ Luca Carli $^{4}$ Enrico Vaccari ${ }^{4}$, Ulug Murat ${ }^{5}$, Wilfried Feichtinger ${ }^{4}$

${ }^{1}$ IVF Unit, Cervesi Hospital Cattolica, Cattolica (RN), Italy

2Section of Clinical Biochemistry and Molecular Genetics, Department of Biomolecular Sciences, University of Urbino Carlo Bo, Urbino (PU), Italy

${ }^{3}$ IVF Unit, Versilia Hospital, Lido di Camaiore (LU), Italy

${ }^{4}$ Wunschbaby Zentrum Feichtinger, Wien, Austria

${ }^{5}$ IVF Unit, Ota Jinemed Hospital, Istanbul, Turkey

\begin{abstract}
Objective: The aim of this study was to evaluate the efficacy of a non-expensive, easy and fast technique (direct micro swim-up) for sperm preparation in intracytoplasmic sperm injection (ICSI) treatments without the use of centrifuge.

Methods: We carried out a multicentric study in which a total of 140 ICSI-cycles were included. Sibling oocytes were divided into two groups according to semen preparation procedures: group $A$, discontinuous gradients (DG) (oocytes $n=668$ ), and group $B$, direct micro swim-up (MSU) (oocytes $n=660$ ). We analyzed differences in some key performance indicators.

Results: Fertilization rates were not statistically different between the DG and MSU groups (76.0\% vs. $81.8 \%$, respectively, $p=0.248$ ); while significant differences were found in blastulation rates per fertilized oocytes $(41.7 \%$ vs. $58.5 \%, p=0.009)$, blastulation rates per D3 embryos ( $46.1 \%$ vs. $63.7 \%, p=0.045)$, and pregnancy rates $(25.8 \%$ vs. $41.9 \%, p=0.045)$. The abortion rate was reduced in the MSU group as compared to DG, but not in a significant manner (12.9\% vs. $29.4 \%, p=0.161)$.

Conclusion: The MSU procedure has the advantage of reducing costs, time and mismatches, while ensuring comparable, and in some cases, better results than DG treatments. This technique can therefore be used as an alternative method to other conventional semen treatments.
\end{abstract}

Keywords: Sperm treatment, centrifugation, swim-up, pregnancy rate, blastulation rate, DNA damage

\section{INTRODUCTION}

Many methods have been developed for semen sample preparation for in vitro fertilization (IVF) treatments according to semen quality parameters (concentration, motility, morphology). The procedures include, as major techniques, simple washing, direct swim-up, and discontinuous density gradients (World of Health Organization, 2010).

To perform these procedures, a laboratory must be equipped with specific materials and media that increase the costs of the entire IVF treatment. Moreover, all the procedures require centrifugations varying in speed and time, and the movement of the samples from one tube to another thus increasing the risk of mismatches.

A wide variability in the techniques used for semen treatments leads to the need to evaluate the potential benefits and risks of each procedure, in terms of (i) sperm function, embryo development and treatment outcome; (ii) technical complexity and risk of sample mismatches; (iii) material costs and time of the procedure.

Regarding sperm function maintenance following the two most conventional methods of sperm preparation, namely swim-up (SU) and discontinuous gradients (DG), there are different opinions in the literature. Some authors reported a higher recovery rate of total motile, progressive motile and viable sperm cells after DG than after SU (Ding et al., 2000; Ricci et al., 2009). Other benefits of DG are the higher percentage of morphologically normal spermatozoa (Prakash et al., 1998; Hammadeh et al., 2001) and the lower deformity rate and DNA fragmentation index (Xue et al., 2014). In contrast, other authors reported that the proportion of fast spermatozoa was enhanced in SU preparations (Chantler et al., 2004) and that spermatozoa reported less vacuolizations (Monqaut et al., 2011).

Another topic of debate concerns the use of the centrifuge and the formation of reactive oxygen species (ROS) during semen treatment. DG centrifugation may be detrimental to sperm DNA integrity, especially in semen samples from infertile men with impaired motility and morphology (Zini et al., 2000). These samples generate high pathological ROS levels (because of morphologically abnormal sperm) and have a lower total antioxidant capacity (TAC) than seminal plasma from fertile men (Benedetti et al., 2012). Consequently, serial centrifugations can result in augmented ROS generation and further loss of sperm function, probably due to the enhanced DNA denaturation during centrifugation.

To assess the best procedure for semen treatment, one must also consider the time of centrifugation. Some authors (Shekarriz et al., 1995) reported that the time of centrifugation is more important than $\mathrm{g}$-force for inducing ROS formation in semen, recommending a shorter centrifugation time in the preparation of sperm for assisted reproductive techniques.

Concerning pregnancy rates in intracytoplasmic sperm injection (ICSI) cycles, some authors reported values of $46.2 \%$ and $57.1 \%$ for SU and DG, respectively (Borges et al., 2013), while other papers reported rates of $21.1 \%$ and $33.3 \%$ (Van der Zwalmen et al., 1991) or 33.3\% and $32.8 \%$ (Hammadeh et al., 2001), respectively. These results do not show a conclusion regarding fertilization and pregnancy rates in SU and DG procedures.

In this study, we used a simple, fast and non-expensive technique for semen preparation in ICSI cycles, namely direct micro swim-up (MSU), as recently reported (Palini et al., 2016). MSU is a safe treatment regarding the presence of microbiological contaminants; indeed, we demonstrated that sample fractions obtained after the MSU procedure and used for ICSI insemination were free 
of contaminants even in semen samples positive for sexual pathogens (Palini et al., 2016). Despite this evidence, inclusion criteria for the present study were negative urethral and vaginal swabs, confirmed before treatment, and negative infection tests ( $\mathrm{HIV}, \mathrm{HCV}, \mathrm{HbsAg}, \mathrm{HbcAb}$, VDRL-TPHA).

The aim of the present study was to compare MSU with DG procedures in terms of fertilization rates (FR), blastulation rates (BR), pregnancy rates (PR), and abortion rates (AR).

\section{MATERIALS AND METHODS}

This is a multicentric study involving four Assisted Reproduction Clinics in Europe. The study was approved by an Internal Review Board (IRB) of each participating center. All the four clinics used the same media, procedures and incubators.

We retrospectively evaluated 140 ICSI cycles performed from October 2014 to June 2015 involving 140 couples (women aged $35 \pm 4$ years, men aged $38 \pm 3$ years) undergoing IVF treatments due to male $(n=52)$, female $(n=20)$, multifactorial $(n=8)$, or idiopathic $(n=60)$ infertility. The inclusion criteria were as follows: negative urethral and vaginal swabs confirmed before treatment, negative infection tests (HIV, HCV, HbsAg, HbcAb, VDRLTPHA) for couples, at least 8 mature oocytes retrieved at pickup, oocyte insemination with ICSI technique, only fresh elective single embryo transfer (eSET) at blastocyst stage, and sperm concentration $\geq 1 \times 10^{6} / \mathrm{ml}$.

In each cycle, we divided sibling oocyte populations into two groups according to the technique used for sperm preparation for the ICSI: group A, discontinuous gradients (DG), and group B, direct micro swim-up (MSU) (Figure 1).

The study was carried out in accordance with the Code of Ethics of the World Medical Association (Declaration of Helsinki) for experiments involving humans following the Internal Review Board's approval (granted September 2014).

\section{Semen sample collection}

All semen samples were collected by masturbation (three to five days of ejaculatory abstinence) about one hour before insemination, to reduce the exposure time of spermatozoa to seminal plasma. After fluidification for $10-20$ minutes at $37^{\circ} \mathrm{C}$, semen samples were evaluated according to WHO criteria (2010). Semen with high viscosity were pretreated with the addiction of an equal volume of medium (G Gamete ${ }^{\mathrm{TM}}$ Vitrolife).

\section{DG procedure}

DG procedure was performed using three different gradient concentrations $(95 \%, 70 \%, 50 \%)$ prepared with a density gradient solution (Spermient K-SISP COOK) and a buffer medium (G MOPS ${ }^{\mathrm{TM}}$ Vitrolife). The $95 \%$ layer $(0.5 \mathrm{ml})$ was transferred into a $15-\mathrm{ml}$ conical centrifuge tube (BD Falcon $\left.{ }^{\mathrm{TM}}\right)$ and then the other layers $(0.5 \mathrm{ml})$ were gently released above the previous gradient. A volume of semen sample $(0.5 \mathrm{ml})$ was then placed on the top of the upper layer and the tube was centrifuged for 10 minutes at 1200rpm.

For each DG procedure, we used two $15 \mathrm{ml}$ conical tubes. After centrifugation, the lower layer was recovered in a sterile new tube and the pellet was resuspended with $5 \mathrm{ml}$ of $\mathrm{G}$ Gamete ${ }^{\mathrm{TM}}$ Vitrolife for a subsequent centrifugation at 1200rpm for 5 minutes.

The supernatant was then removed and the pellet was submitted to swim-up by gently overlaying $1 \mathrm{ml}$ of $\mathrm{G}$ Gamete $^{\mathrm{TM}}$ Vitrolife. The tube was inclined at an angle of 45 degrees and left in the incubator for at least 40 minutes under controlled atmosphere conditions $\left(6 \% \mathrm{CO}_{2}, 20 \%\right.$ $\mathrm{O}_{2}$ ). After incubation, the supernatant was aspirated and transferred into a sterile new $15 \mathrm{ml}$ conical tube placed in the incubator until the time of injection. Sperm analysis was done using a sample fraction according to WHO criteria (2010).

\section{MSU procedure}

After semen evaluation, about $1 \mathrm{ml}$ was recovered in a sterile $15 \mathrm{ml}$ conical tube and was submitted to a direct swim-up by gently overlying $1 \mathrm{ml}$ of $\mathrm{G}$ Gamete $^{\mathrm{TM}}$ Vitrolife. The tube was inclined at an angle of 45 degrees and left in the incubator until the time of injection under controlled atmospheric conditions $\left(6 \% \mathrm{CO}_{2}, 20 \% \mathrm{O}_{2}\right)$. In this procedure, the use of the centrifuge was not expected. Sperm analysis was done using a fraction of the direct swim-up semen according to WHO criteria (2010). MSU procedure was performed at ICSI time according to the methods recently published (Palini et al., 2016).

\section{Oocyte retrieval and embryo transfer}

Ultrasound-guided oocyte retrieval was performed 36 hours after human chorionic gonadotropin (HCG) injection. Cumulus cells were removed 3 hours after pickup and the insemination procedure was performed 40 hours after HCG administration. Sibling oocytes were divided into two equal groups and inseminated by the same operator using spermatozoa obtained by DG and MSU. Approximately 1620 hours after insemination, fertilization was confirmed by the presence of two pronuclei and the extrusion of the second polar body (Istanbul Consensus 2011).

The embryos were cultured in a humidified atmosphere with $6 \% \mathrm{CO}_{2}, 5 \% \mathrm{O}_{2}$, and remaining nitrogen; and on Day 3 (D3) they were classified according to the grading proposed by the ALPHA and ESHRE societies (Istanbul Consensus) (2011).

Elective fresh single embryo transfer (eSET) was performed on Day 5 (D5) after insemination; blastocyst cryopreservation was performed on Day 6 (D6) after insemination by Vitrification technique. D5 and D6 embryos were classified by all the Clinics according to a revisited Gardner grading (Veeck \& Zaninovic, 2003).

Pregnancy was confirmed by serum beta HCG test 14 days after transfer.

\section{Statistical analysis}

Differences between DG and MSU groups' numbers of mature and fertilized oocytes, numbers of D3 and D5/D6 embryos, and numbers of transferred and cryopreserved embryos were assessed by the t-test for paired data. Differences in fertilization and blastulation rates were assessed by the $t$-test for paired data after arcsine transformation of the data. Differences regarding pregnancy and abortion rates were evaluated by the Chisquare test. Probability values $\leq 0.05$ were accepted.

\section{RESULTS}

To test the effectiveness of MSU as compared to DG, the following indicators were considered: fertilization rate $(F R)$, blastulation rate (BR), pregnancy rate (PR), and abortion rate (AR). Results are summarized in Table 1.

Differences in fertilization rates were not significant between the DG and MSU groups $(76.0 \%$ vs. $81.8 \%$, respectively, $p=0.248$ ); while significant differences were found for blastulation rates per fertilized oocyte $(41.7 \%$ vs. $58.5 \%, p=0.009)$, blastulation rates per D3 embryos ( $46.1 \%$ vs. $63.7 \%, p=0.045)$, and pregnancy rates $(25.8 \%$ vs. $41.9 \%, p=0.045)$. The abortion rate was reduced in the MSU group as compared to the DG, but not in a significant 


\section{ICSI cycles}

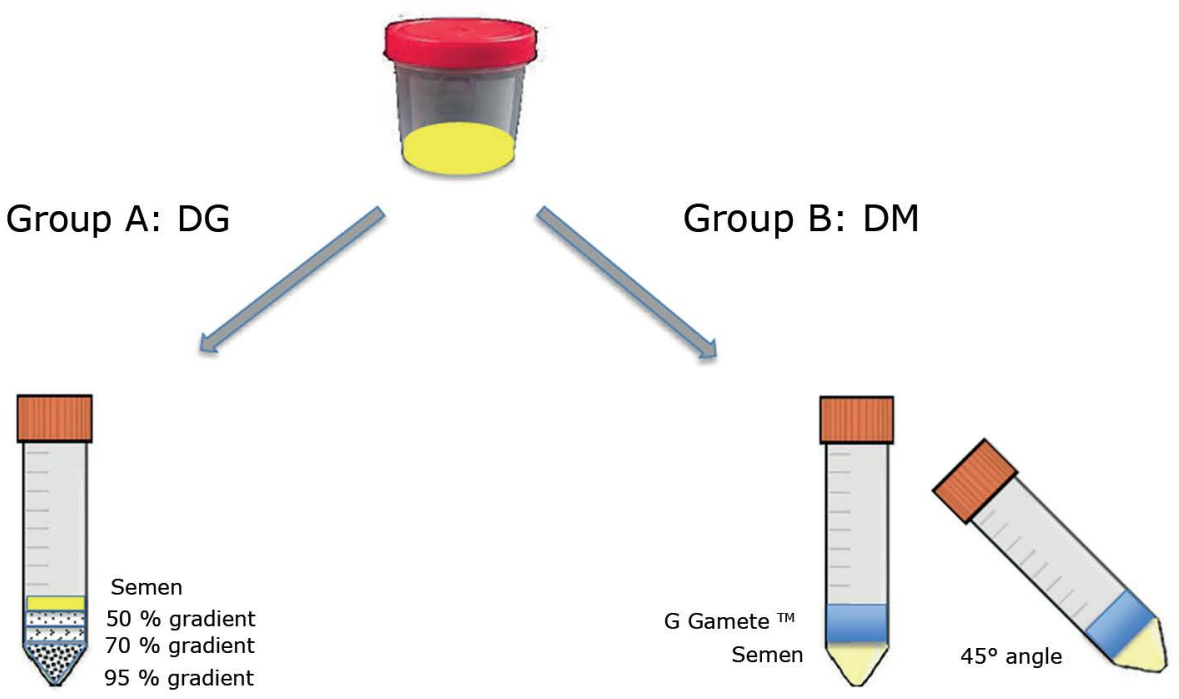

First centrifugation: $1200 \mathrm{rpm}$ 10min Second centrifugation: 1200rpm 5min

NO centrifugation

\section{ICSI oocytes}

660 ICSI oocytes

Figure 1. Study design.

Table 1. Differences in fertilization rate (FR), blastulation rate (BR), pregnancy rate (PR), and abortion rate (AR) between DG and MSU groups.

\begin{tabular}{|l|c|c|c|}
\hline & DG & MSU & P value \\
\hline Mature oocytes & 668 & 660 & 0.660 \\
\hline Fertilized oocytes & 508 & 540 & 0.174 \\
\hline D3 embryos & 460 & 496 & 0.132 \\
\hline D5/D6 embryos & 212 & 316 & $<0.001$ \\
\hline Transferred D5/D6 embryos & 66 & 74 & 0.666 \\
\hline Cryopreserved D5/D6 embryos & 82 & 168 & $<0.001$ \\
\hline FR (\%) & $76.0(508 / 668)$ & $81.8(540 / 660)$ & 0.248 \\
\hline BR per fertilized oocyte (\%) & $41.7(212 / 508)$ & $58.5(316 / 540)$ & 0.009 \\
\hline BR per D3 embryo (\%) & $46.1(212 / 460)$ & $63.7(316 / 496)$ & 0.045 \\
\hline PR (\%) & $25.8(17 / 66)$ & $41.9(31 / 74)$ & 0.045 \\
\hline AR (\%) & $29.4(5 / 17)$ & $12.9(4 / 31)$ & 0.161 \\
\hline
\end{tabular}

manner $(12.9 \%$ vs. $29.4 \%, p=0.161)$, probably due to the small size of the population. Differences in the numbers of frozen D5/D6 embryos were significant between DG and MSU (82 vs. 168, $p<0.001)$.

\section{DISCUSSION}

A wide variety of sperm preparation protocols are currently available in IVF treatments. The most used techniques are density gradient (DG) centrifugation and swim-up (SU) in cases of normospermia or moderate oligoasthenoteratozoospermia. In patients with severe oligoasthenoteratozoospermia, strategies involving high speed centrifugation are used to recover spermatozoa from all semen samples.

The presence of a wide variability in the techniques used for semen treatments leads to a need to evaluate the potential benefits and risks of each procedure. The literature does not show a conclusion regarding 
fertilization and pregnancy rates in SU and DG procedures but it demonstrates benefits in favor of SU in terms of DNA damage and ROS production (Zini et al., 2000).

The MSU technique used in the present study is similar to that of SU, but it offers the advantage of using no centrifugation, and still enabling an efficient selection of spermatozoa by both the SU into the conical tube and the MSU in polyvinylpyrrolidone (PVP), providing a gentler and safer treatment. The present study not only demonstrates the greater reproducibility of the results with MSU when compared with the DG, but what stands out is the possibility to use a non-invasive method on semen treatment with statistically better results for embryo development and pregnancy, that can potentially further increase, considering the cumulative pregnancy with subsequent embryo warming. Differences in the number of frozen D5/D6 embryos were significant between DG and MSU, probably due to the better quality of embryos obtained from the MSU group.

One explanation for this could be found in the better health status of the embryos obtained from spermatozoa that have suffered less stress during the treatment; in fact, paternal contribution to embryo genome could be evident, especially in blastocyst development (Simon et al., 2014). Evidence of this is given by our preliminary results (data not shown) that demonstrate, through an array of Comparative Genetic Hybridization (aCGH) analysis, that blastocysts belonging to the MSU group have a better euploid status than those from the DG group. More data is needed to confirm this hypothesis.

Furthermore, we must take into consideration the potential epigenetic modifications that serial centrifugations and ROS production can have on spermatozoa, which are at potentially greater risk because of their extended processing specially during the DG technique. One study demonstrated a relationship between differential levels of sperm DNA methylation and pregnancy rate in IVF treatments (Benchaib et al., 2005); a later study reported correlations between sperm DNA methylation and semen quality (Houshdaran et al., 2007). Because of this, spermatozoa may be carriers of epigenetic modifications that may open another "epigenetic programming window" in the embryo, introducing changes in DNA imprint (Miller et al., 2014).

It may be noted that the proposed MSU technique, while being safer, does not allow sperm capacitation (Palini et al., 2016). However, our data shows that sperm activation through immobilization before ICSI can be enough to enable the fertilization process. Indeed, sperm plasma membrane damage has been described as a necessary process prior to ICSI, as it plays a key role in oocyte activation caused by spermatozoa (Dozortsev et al., 1995; Palermo et al., 1996).

The SU procedure and the subsequent MSU also allowed to avoid the risk of mismatches, because they reduced the number of conical tubes used for the treatment of seminal samples. By comparing the two procedures used in the present study, a total of 3 tubes were used in DG, while only one after semen fluidification in MSU. Despite this, the presence of two operators was always required for a double check during the transition from the collecting container to the conical tube and from the conical tube to the ICSI dish (Magli et al., 2008).

Another point in favor of SU and MSU treatments is the reduction of time and costs. In fact, sperm collection and $\mathrm{SU}$ can be performed in proximity of the ICSI insemination time, avoiding the risk of reduced DNA longevity. Unnecessary incubation of spermatozoa prior to artificial insemination or in vitro fertilization should be avoided, since sperm DNA longevity is significantly reduced after ex vivo sperm handling, and sperm DNA longevity after DG is lower when compared to sperm recovered from noncentrifuged semen (Gosálvez et al., 2014). When using MSU, 30 minutes are sufficient for SU and 3 minutes for micro swim-up in PVP.

By using MSU, it is also possible to reduce costs since less consumables (such as pipettes and tubes), culture medium and gradient solution are required. Furthermore, although the insemination technique (ICSI) remains expensive for the need of a micromanipulator, it would be possible to insert the MSU procedure into an IVF low cost program, thus reducing the total cost of the treatment (Van Blerkom et al., 2014; Teoh et al., 2014).

In conclusion, procedures that minimize excessive manipulation of sperm and centrifugation should be used in IVF treatment as a valid alternative to improve the outcome. This non-invasive technique enables to obtain embryos with a physiological and probably better euploid status, although further data is needed to prove it. By using the MSU procedure, it is also possible to reduce costs and mismatch errors because less steps are required for semen treatment, facilitating the workflow in an IVF laboratory.

\section{FUNDING}

This study did not receive any specific grant from funding agencies in the public, commercial, or non-profit sectors.

\section{ACKNOWLEDGEMENTS}

The authors wish to thank Dr. Mirka ES Scarbaci for her technical help and support.

\section{CONFLICT OF INTERESTS}

The authors have no conflicts of interest to report.

\section{CORRESPONDING AUTHOR}

Simone Palini

IVF Unit - Marche nord Hospital

Pesaro (PU) - Italy

E-mail: simonepalini@yahoo.it

\section{REFERENCES}

Alpha Scientists in Reproductive Medicine and ESHRE Special Interest Group of Embryology. The Istanbul consensus workshop on embryo assessment: proceedings of an expert meeting. Hum Reprod. 2011;26:1270-83. PMID: 21502182 DOI: 10.1093/humrep/der037

Benchaib $M$, Braun V, Ressnikof D, Lornage J, Durand $P$, Niveleau A, Guérin JF. Influence of global sperm DNA methylation on IVF results. Hum Reprod. 2005;20:768-73. PMID: 15640258 DOI: 10.1093/humrep/deh684

Benedetti S, Tagliamonte MC, Catalani S, Primiterra M, Canestrari F, De Stefani S, Palini S, Bulletti C. Differences in blood and semen oxidative status in fertile and infertile men, and their relationship with sperm quality. Reprod Biomed Online. 2012;25:300-6. PMID: 22818093 DOI: 10.1016/j.rbmo.2012.05.011

Borges E Jr, Setti AS, Vingris L, Figueira Rde C, Braga DP, Iaconelli A Jr. Intracytoplasmic morphologically selected sperm injection outcomes: the role of sperm preparation techniques. J Assist Reprod Genet. 2013;30:849-54. PMID: 23547020 DOI: $10.1007 / s 10815-013-9989-x$ 
Chantler E, Abraham-Peskir J, Roberts C. Consistent presence of two normally distributed sperm subpopulations within normozoospermic human semen: a kinematic study. Int J Androl. 2004;27:350-9. PMID: 15595954 DOI: 10.1111/j.1365-2605.2004.00498.x

Ding DC, Liou SM, Huang LY, Liu JY, Wu GJ. Effects of four methods of sperm preparation on motion characteristics and nitric oxide concentration in laboratory-prepared oligospermia. Zhonghua Yi Xue Za Zhi (Taipei). 2000;63:822-7. PMID: 11155759

Dozortsev D, Rybouchkin A, De Sutter P, Dhont M. Sperm plasma membrane damage prior to intracytoplamsic sperm injection: a necessary condition for sperm nucleus decondensation. Hum Reprod. 1995;10:2960-4. PMID: 8747054 DOI: http://dx.doi.org/10.1093/oxfordjournals. humrep.a135829

Gosálvez J, Johnston S, López-Fernández C, Gosálbez A, Arroyo F, Fernández, JL, Gálvarez J. Sperm fractions obtained following density gradient centrifugation in human ejaculates show differences in sperm DNA longevity. Asian Pac J Reprod. 2014;3:116-20. https://doi.org/10.1016/S2305-0500(14)60014-2

Hammadeh ME, Kühnen A, Amer AS, Rosenbaum $P$, Schmidt W. Comparison of sperm preparation methods: effect on chromatin and morphology recovery rates and their consequences on the clinical outcome after in vitro fertilization embryo transfer. Int J Androl. 2001;24:360-8. PMID: 11737417 DOI: http://dx.doi.org/10.1046/j.1365-2605.2001.0317a.x

Houshdaran S, Cortessis VK, Siegmund K, Yang A, Laird PW, Sokol RZ. Widespread epigenetic abnormalities suggest a broad DNA methylation erasure defect in abnormal human sperm. PLoS One. 2007;2:e1289. PMID: 18074014 DOI: 10.1371/journal.pone.0001289

Magli MC, Van den Abbeel E, Lundin K, Royere D, Van der Elst J, Gianaroli L; Committee of the Special Interest Group on Embryology. Revised guidelines for good practice in IVF laboratories. Hum Reprod. 2008;23:1253-62. PMID: 18375408 DOI: $10.1093 /$ humrep/den068

Miller D. Sperm RNA as a Mediator of Genomic Plasticity. Adv Biol. 2014;2014:179701. DOI: 10.1155/2014/179701

Monqaut AL, Zavaleta C, López G, Lafuente R, Brassesco M. Use of high magnification microscopy for the assessment of sperm recovered after two different sperm processing methods. Fertil Steril. 2011;95:277-80. PMID: 20674902 DOI: $10.1016 /$ j.fertnstert.2010.06.071

Palermo G, Schlegel P, Colombero L, Zaninovic N, Moy $\mathrm{F}$, Rosenwaks Z. Aggressive sperm immobilization prior to intracytoplasmic sperm injection with immature spermatozoa improves fertilization and pregnancy rates. Hum Reprod. 1996;11:1023-9. PMID: 8671384 DOI: http://dx.doi.org/10.1093/oxfordjournals.humrep. a019290

Palini S, Primiterra M, De Stefani S, Pedna MF, Sparacino M, Farabegoli P, Benedetti S, Bulletti C, Sambri V. A new micro swim-up procedure for sperm preparation in ICSI treatments: preliminary microbiological testing. JBRA Assist Reprod. 2016;20:94-8. PMID: 27584599 DOI: 10.5935/1518-0557.20160023
Prakash P, Leykin L, Chen Z, Toth T, Sayegh R, Schiff $I$, Isaacson $K$. Preparation by differential gradient centrifugation is better than swim-up in selecting sperm with normal morphology (strict criteria). Fertil Steril. 1998;69:722-6. PMID: 9548164 DOI: http://dx.doi.org/10.1016/S0015-0282(98)00002-8

Ricci G, Perticarari S, Boscolo R, Montico M, Guaschino $S$, Presani G. Semen preparation methods and sperm apoptosis: swim-up versus gradient-density centrifugation technique. Fertil Steril. 2009;91:632-8. PMID: 18206147 DOI: $10.1016 /$ j.fertnstert.2007.11.068

Shekarriz M, DeWire DM, Thomas AJ Jr, Agarwal A. A method of human semen centrifugation to minimize the iatrogenic sperm injuries caused by reactive oxygen species. Eur Urol. 1995;28:31-5. PMID: 8521891

Simon L, Murphy K, Shamsi MB, Liu L, Emery B, Aston KI, Hotaling J, Carrell DT. Paternal influence of sperm DNA integrity on early embryonic development. Hum Reprod. 2014;29:2402-12. PMID: 25205757 DOI: $10.1093 /$ humrep/deu228

Teoh PJ, Maheshwari A. Low-cost in vitro fertilization: current insights. Int J Womens Health. 2014;6:817-27. PMID: 25187741 DOI: 10.2147/IJWH.S51288

Van Blerkom J, Ombelet W, Klerkx E, Janssen M, Dhont N, Nargund G, Campo R. First births with a simplified culture system for clinical IVF and embryo transfer. Reprod Biomed Online. 2014;28:310-20. PMID: 24456702 DOI: 10.1016/j.rbmo.2013.11.012

Van der Zwalmen P, Bertin-Segal G, Geerts L, Debauche C, Schoysman R. Sperm morphology and IVF pregnancy rate: comparison between Percoll gradient centrifugation and swim-up procedures. Hum Reprod. 1991;6:581-8. PMID: 1918311 DOI: http://dx.doi.org/10.1093/oxfordjournals. humrep.a137383

Veeck LL, Zaninovic N. An Atlas of Human Blastocysts. Boca Raton: CRC Press; 2003.

World Health Organization, Department of Reproductive Health and Research. Laboratory manual for the examination and processing of human semen. 5th ed. Geneva: World Health Organization; 2010.

Xue X, Wang WS, Shi JZ, Zhang SL, Zhao WQ, Shi WH, Guo BZ, Qin Z. Efficacy of swim-up versus density gradient centrifugation in improving sperm deformity rate and DNA fragmentation index in semen samples from teratozoospermic patients. J Assist Reprod Genet. 2014;31:1161-6. PMID: 25015033 DOI: $10.1007 / \mathrm{s} 10815-014-0287-\mathrm{z}$

Zini A, Nam RK, Mak V, Phang D, Jarvi K. Influence of initial semen quality on the integrity of human sperm DNA following semen processing. Fert Steril. 2000;74:824-7. PMID: 11020532 DOI: http://dx.doi.org/10.1016/S0015-0282(00)01495-3 